\title{
Síndrome de Alagille Alagille syndrome
}

\author{
Dra. Mirta Ciocca $a^{a}$ y Dr. Fernando Álvarez
}

\section{RESUMEN}

El síndrome de Alagille (SA) es una enfermedad autosómica dominante multisistémica, con expresión variable. Las principales manifestaciones son: colestasis crónica, enfermedad cardíaca congénita, embriotoxón posterior en el examen ocular, fenotipo facial característico y vértebras en alas de mariposa. Es ocasionado por mutaciones en el JAGGED1 (más del 90\%) y en el gen $\mathrm{NOTCH}$. El diagnóstico diferencial incluye: infecciones, enfermedades genético-metabólicas, atresia biliar, causas idiopáticas. La colestasis, el prurito intenso y los xantomas tienen indicación de coleréticos (ácido ursodesoxicólico) y otras medicaciones (colesteramina, rifampicina, naltrexona). En ciertos casos, la derivación biliar parcial externa ha resultado eficaz. El trasplante hepático está indicado en niños con cirrosis e insuficiencia hepática.

Palabras clave: sindrome de Alagille, colestasis, hipercolesterolemia, prurito.

\section{SUMMARY}

Alagillesyndrome(AS) is a multisystemic disease autosomal dominant, with variable expression. The major clinical manifestations are: chronic cholestasis, congenital heart disease, posterior embryotoxon in the eye, characteristic facial phenotype, and butterfly vertebrae. AS is caused by mutations in JAGGED1 (more than 90\%) and in $\mathrm{NOTCH}$ 2. Differential diagnosis include: infections, genetic-metabolic diseases, biliary atresia, idiopathic cholestasis. Cholestasis, pruritus and xanthomas have been successfully treated with choleretic agents (ursodeoxycholic acid) and other medications (cholestyramine, rifampin, naltrexone). In certain cases, partial external biliary diversion has also proved successful. Liver transplantation is indicated in children with cirrhosis and liver failure.

Key words: Alagille syndrome, cholestasis, hypercholesterolemia, pruritus

http:/ /dx.doi.org/10.5546/aap.2012.509

\section{INTRODUCCIÓN}

El síndrome de Alagille (SA), conocido también como síndrome de Alagille-Watson y displasia arteriohepática, es una enfermedad multisistémica autosómica dominante, ocasionada por defectos en la vía de señalización del receptor Notch. Los primeros casos fueron publicados por Alagille y cols., en 1969. Con el adve- nimiento del diagnóstico molecular, se considera que su frecuencia es de aproximadamente 1/30 000. La mayoría de los casos (aproximadamente el $97 \%$ ) es ocasionada por haploinsuficiencia del gen JAGGED1 (JAG1) sobre el brazo corto del cromosoma 20, debido predominantemente a mutaciones o deleciones del locus. En más de la mitad de los casos se trata de mutaciones de novo, inexistentes en los genes de los padres. Un pequeño porcentaje $(<1 \%)$ es causado por mutaciones en el gen NOTCH2, asociándose a malformaciones renales. ${ }^{1-7}$

\section{Características clínicas}

Tradicionalmente, el SA fue considerado en niños con colestasis neonatal. Con el tiempo, se estableció el denominado "Criterio Clásico" (Tabla 1), con 5 anormalidades clínicas principales: colestasis debida a pobreza de conductos biliares (disminución del número de conductos biliares en relación al número de espacios porta, en la biopsia hepática), enfermedad cardíaca congénita, anormalidades esqueléticas, alteraciones oculares y fenotipo facial característico (Figuras 1 y 2). Las manifestaciones clínicas son muy variables. Los estudios familiares han demostrado un amplio espectro de ellas, desde enfermedades cardíacas, renales o hepáticas graves hasta solo expresiones subclínicas. ${ }^{1-4}$

\section{Enfermedad hepática}

El compromiso hepático se caracteriza por colestasis crónica en alrededor del $95 \%$ de los casos, mayoritariamente durante el período neonatal. Existe hepatoesplenomegalia, hiperbilirrubinemia conjugada, hipercolesterolemia, hipertrigliceridemia, y ácidos biliares y enzimas hepáticos aumentados. La hipercolesterolemia del 
SA suele ser notablemente elevada, sin embargo, como el nivel sanguíneo de colesterol se asocia con la lipoproteína- $X$, resiste la oxidación y protege al paciente de la aterosclerosis.

Además, suelen presentar retraso del crecimiento, prurito y xantomas. El prurito se halla entre los más graves debidos a afecciones hepáticas, suele ser invalidante y se presenta a partir del segundo semestre de vida. Los xantomas suelen observarse con una concentración de colesterol superior a $500 \mathrm{mg} / \mathrm{dl}$ y se localizan sobre la superficie extensora de los dedos, pliegues palmares, nuca, orejas, fosa poplítea, glúteos y alrededor de los pliegues inguinales. Se incrementan en número durante los primeros años de vida y pueden desaparecer posteriormente, cuando la colestasis mejora. Su repercusión es cosmética, además de interferir con la función motora fina, aunque sin ocasionar dolor.

TABLA 1. Frecuencia de presentación de anormalidades características del sindrome de Alagille

\begin{tabular}{lc}
\hline Características clínicas & Presencia en \% \\
\hline Pobreza de vías biliares & $75-100 \%$ \\
Colestasis crónica & $89-100 \%^{*}$ \\
Soplo (auscultación torácica) & $85-98 \%$ \\
Anomalías vertebrales & $33-87 \%$ \\
Facies & $75-95 \%$ \\
Anomalías oculares & $56-88 \%$ \\
Anomalías renales & $19-73 \%$ \\
\hline
\end{tabular}

* En la mayoría de las series publicadas, la colestasis es más frecuente que el patrón histológico con pobreza de vías biliares intrahepáticas. Esta constatación es otra evidencia de una distribución heterogénea de la presencia o ausencia de canales biliares en el hígado.

FIgURA 1. Vértebras en "alas de mariposa" (flecha) en una radiografía de columna dorsal

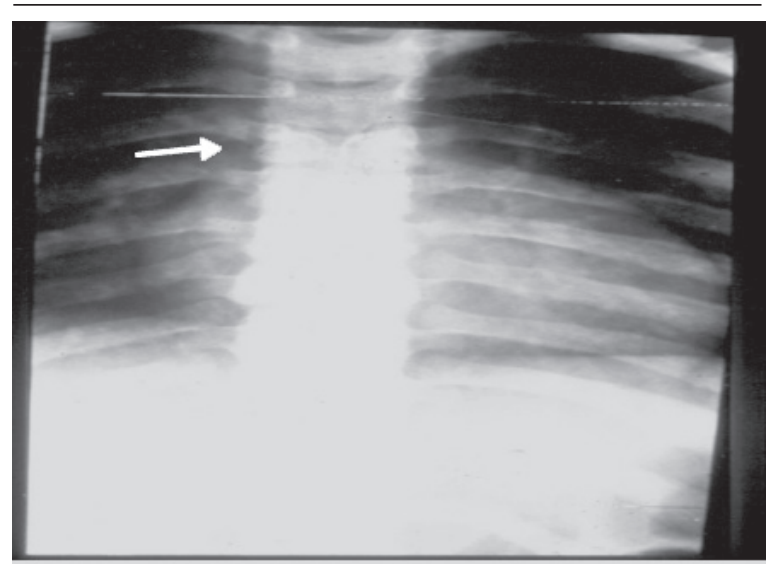

La biopsia hepática ya no es obligatoria si existe colestasis. Típicamente, muestra pobreza de conductos biliares intrahepáticos, aunque en neonatos suele observarse proliferación ductal con inflamación portal, hecho que puede conducir al diagnóstico erróneo de atresia biliar (AB). Conocer esta posibilidad es muy importante, pues la $\mathrm{AB}$ puede mejorar con un procedimiento de Kasai, el cual no favorecería a los niños con SA.

La colestasis suele intensificarse hasta la edad escolar y luego, en algunos niños, mejora o permanece estable. La distribución heterogénea de la escasez de vías biliares interlobulares en el hígado provoca atrofia de las regiones sin vía biliar e hipertrofia de las que las poseen, lo cual confiere un aspecto nodular en la ecografía hepática. Estos nódulos con tejido hepático normal pueden explicar la mejoría de la colestasis. La evolución hacia una enfermedad hepática progresiva, cirrosis y fallo hepático, con indicación de trasplante hepático, acontece en el 15\% de los casos. Existen numerosas descripciones acerca del desarrollo de carcinoma hepatocelular en pacientes con SA. Aunque es una complicación infrecuente, tiene elevada morbimortalidad. ${ }^{8-11}$

\section{Enfermedad cardíaca}

El compromiso cardíaco está presente en más del $90 \%$ de los niños. La cardiopatía congénita más frecuente es la estenosis pulmonar periférica $(67 \%)$. Otras malformaciones, con frecuencia decreciente, son: tetralogía de Fallot (16\%), defecto septal ventricular, defecto septal auricular, estenosis aórtica y coartación de aorta. La presencia de alguna de estas cardiopatías congénitas puede

FIgURA 2. Características faciales de la madre y sus dos hijos con sindrome de Alagille. Entre otras características presentan: a) frente prominente, $b$ ) puente nasal ancho $y$ deprimido, c) mentón puntiagudo

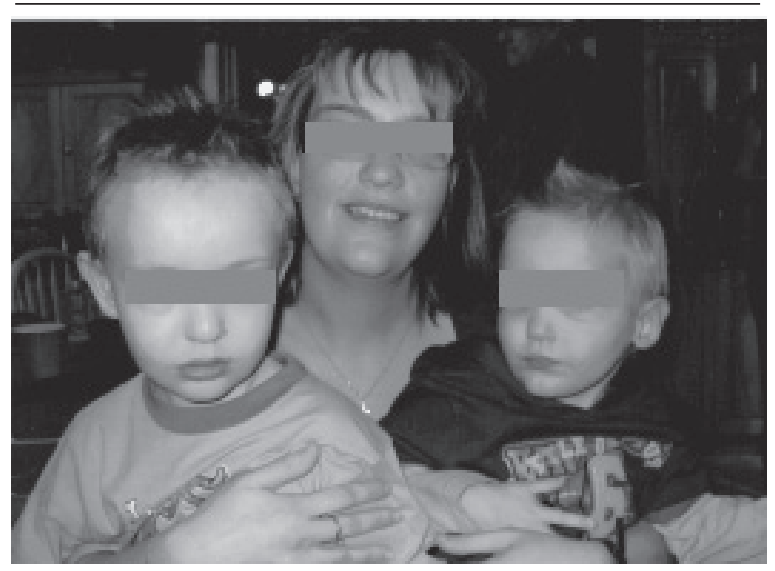


ser la única manifestación de la enfermedad. Una cardiopatía compleja es el indicador más significativo de mortalidad temprana del paciente con SA (Tabla 2). ${ }^{1,4,11-13}$

\section{Manifestaciones oftalmológicas}

El hallazgo más frecuente es el embriotoxón posterior (78-89\%), que consiste en una banda prominente, blanca, situada en el ángulo de la cámara anterior del ojo. Se identifica con lámpara de hendidura y se detecta en el $8-15 \%$ de la población normal. Otras afecciones vinculadas con el SA son: microcórnea, keratocono, distrofia macular congénita, cámara anterior superficial, exotropia, queratopatía en banda y cataratas. La hipopigmentación difusa del fundus retiniano puede ocurrir en hasta un $57 \%$ de los pacientes, el moteado del epitelio pigmentario retiniano en el $33 \%$ y anomalías de la papila en el $76 \%$. Nischal et al. hallaron evidencias de drusas en por lo menos un ojo en el $95 \%$ de los pacientes y bilateral en el $80 \%$. La agudeza visual suele ser normal, pero se recomiendan los exámenes oftalmológicos rutinarios destinados a la detección de la progresión de dicho compromiso, como así también diagnosticar glaucoma oportunamente. Menos del 1\% de los niños con SA pueden desarrollar una pérdida visual progresiva y eventualmente una ceguera permanente, probablemente secundaria a anomalías vasculares. ${ }^{4,11,14}$

\section{Anormalidades esqueléticas}

En un $80 \%$ de los pacientes se observan las denominadas vértebras en "alas de mariposa" en la radiografía ántero-posterior, ocasionadas por anomalías en la fusión del arco vertebral anterior. Las anomalías de segmentación vertebral ocurren en diversas enfermedades, pero, en este síndrome, las "alas de mariposa" son de apariencia simé-

TABLA 2. Causa de muerte en pacientes con síndrome de Alagille*

\begin{tabular}{lc}
\hline Complicaciones infecciosas & $9 \%$ \\
Malformación cardíaca & $7,5 \%$ \\
Enfermedad hepática: & $5 \%$ \\
$\quad$ Hipertensión portal: sangrado & \\
$\quad$ Insuficiencia hepática & \\
Lesiones arteriales: & $2,5 \%$ \\
$\quad$ Sistema nervioso central & \\
$\quad$ Periféricas & $1,25 \%$ \\
\hline Insuficiencia renal & \\
\hline * Según fue publicado por Alagille y col. $1987^{3}$. &
\end{tabular}

trica. En general, no tienen repercusión clínica, aunque en raros casos pueden provocar un desplazamiento vertebral y compresión de la médula espinal. Se desconoce la incidencia en la población general, pero se sospecha que es baja. Otras anormalidades incluyen adelgazamiento de la distancia interpeduncular de la columna lumbar, espina bífida oculta y fusión de la vértebra adyacente, hemivértebra y ausencia de la duodécima costilla. Se han comunicado craniosinostosis, sinostosis radiocubital, acortamiento de las falanges distales de los dedos que confiere una apariencia fusiforme. Otra manifestación es la afectación metabólica ósea, evidenciada por osteoporosis y fracturas, que obedecen más al compromiso multiorgánico de la enfermedad y a un estado nutricional subóptimo. La presencia de colestasis crónica puede afectar el metabolismo óseo, conducir a deficiencia de ácidos biliares intestinales e interferir en la absorción de vitaminas y minerales fundamentales para el desarrollo óseo. Un subgrupo de pacientes con SA también presenta acidosis tubular renal, la cual puede, además, incrementar el riesgo de osteomalacia. ${ }^{15-17}$

\section{Características faciales}

Existen características dismórficas leves pero reconocibles, incluidos una frente prominente, ojos hundidos con moderado hipertelorismo, fisuras palpebrales oblicuas ascendentes, puente nasal deprimido, nariz recta con punta bulbosa, orejas grandes, mandíbula prominente y mentón puntiagudo. En algunos niños, la forma de la cara es notablemente triangular. La frente prominente parece ser menos evidente en el adulto, mientras que la protrusión de la mandíbula y el mentón puntiagudo son más obvios. Se ha considerado que el fenotipo facial difiere en relación a si los ojos están o no hundidos. Quienes no presentan ojos hundidos suelen evidenciar fisuras palpebrales oblicuas ascendentes y estrechas, con notable hipertelorismo. ${ }^{1-4,8-11,18}$

\section{Afectación vascular}

Los accidentes cerebrovasculares se han diagnosticado en hasta un 15\% de los pacientes y han sido la causa de muerte en el $34 \%$ de una serie. Las lesiones pueden ser detectadas con una angiorresonancia magnética cerebral. Se han identificado anormalidades de las arterias basilar, carótida y cerebral media, incluido un aspecto vascular compatible con el diagnóstico de enfermedad de Moyamoya. Además se han comunicado anomalías renovasculares y el síndrome de la capa me- 
dia aórtica. Corresponde tener en cuenta que los traumatismos cerebrales menores pueden conducir a hemorragia intracraneana. Lykavieris y cols. han destacado, en pacientes con SA, el riesgo de sangrado al realizar procedimientos invasivos como, por ejemplo, una biopsia hepática. ${ }^{11,19,20}$

\section{Afectación renal}

Las siguientes alteraciones estructurales renales se han descripto en el SA: riñones pequeños y ecogénicos, quistes, obstrucción ureteropiélica, nefropatía tubulointersticial y mesangiolipidosis. Los quistes pueden ir desde los simples, sin consecuencias funcionales, los riñones multiquísticos displásicos, hasta los riñones quísticos que conducen a la insuficiencia renal durante la infancia. Se han comunicado compromiso renal vascular y acidosis tubular renal (en hasta el 74\% de los casos). ${ }^{21,22}$

\section{Retraso del crecimiento}

En una proporción considerable de pacientes se observa retraso del crecimiento. Es probablemente multifactorial, vinculado al defecto primario del JAG1, compromiso esquelético, aporte calórico insuficiente, esteatorrea de origen hepático o pancreático y enfermedad cardíaca significativa. En algunos pacientes se han descripto hipotiroidismo, retraso del desarrollo puberal y, ocasionalmente, falta de sensibilidad a la hormona del crecimiento. ${ }^{8-11,23}$

\section{Afectación pancreática}

Se describe la existencia de insuficiencia pancreática en hasta un $40 \%$ de los pacientes; sin embargo, su evaluación es difícil debido a la esteatorrea secundaria a colestasis. La suplementación oral con enzimas pancreáticas ha resultado beneficiosa en ciertas circunstancias. Además, algunos pacientes han desarrollado diabetes mellitus insulinodependiente..$^{8-11}$

\section{Dificultades del aprendizaje}

Los primeros estudios señalaron la elevada frecuencia de retardo mental en estos pacientes, lo cual puede representar una consideración sesgada. Con tratamiento intensivo, los trastornos del aprendizaje pueden ser comparables con los de la población general, pero parece existir una frecuencia incrementada de retardo motor, que afecta hasta a un $16 \%$ de los casos. Concretamente, las dificultades del aprendizaje son más probables en los casos relacionados con deleciones citogenéticas extensas del cromosoma 20p12.,8-11

\section{Patogenia}

El denominado "Criterio Clásico" de diagnóstico de SA consideró a la pobreza de conductos biliares un patrón fundamental. Actualmente se desconocen los factores que conducen a la disminución de los conductos intrahepáticos. La responsabilidad de la proteína JAG1 en el desarrollo de nuevos conductos en la infancia, no se ha confirmado aún. También se ha observado, en algunos pacientes, reducción en el número de espacios porta y proliferación ductular.

Se desconoce si la variabilidad fenotípica del SA depende de la influencia genotípica. La facies característica planteó la disyuntiva de si se trataba de una malformación primaria o si era consecuencia de la colestasis. Un panel de dismorfologistas sugirió, enfáticamente, que era la consecuencia del genotipo correspondiente.

Otro aspecto muy importante por considerar es el que vincula al SA con una vasculopatía primaria. Las anormalidades vasculares son ampliamente difundidas, y algunas de ellas se vinculan con alteración de la angiogénesis y de la estructura tisular del sistema vascular. Existen evidencias que confirman que la formación de conductos biliares maduros está vinculada con el desarrollo de la red arterial intrahepática. Además, los genes involucrados JAG1 y NOTCH2, son componentes de la vía de señalización del $N O T C H$, la cual desempeña una función primordial en la angiogénesis.

En un $95 \%$ de los pacientes con un convincente diagnóstico clínico de SA se detectan mutaciones en el JAG1 o deleciones del gen 20p12. Hasta la actualidad se han identificado más de 400 mutaciones del JAG1, y aproximadamente el $60 \%$ son de novo. La semejanza fenotípica entre deleción y mutación sugiere que el mecanismo mutacional corresponde a haploinsuficiencia. Una pequeña proporción de pacientes con el criterio clásico de diagnóstico, no presenta ni mutaciones ni deleciones del JAG1 o NOTCH2, lo cual demuestra la probable heterogeneidad genética. ${ }^{11,24-28}$

\section{Diagnóstico diferencial}

La colestasis neonatal representa la expresión clínica más prevalente del SA. En el diagnóstico diferencial corresponde considerar las siguientes entidades: infecciones, enfermedades genético-metabólicas, $\mathrm{AB}$, causas idiopáticas. Las características faciales que permiten sospechar el diagnóstico no siempre son evidentes en las primeras semanas de vida.

La pobreza de conductos biliares descripta en la mayoría de los pacientes con SA se ha observa- 
do además en un grupo diverso de enfermedades como: síndrome de Down, fibrosis quística, infecciones congénitas (CMV, rubéola, sífilis), panhipopituitarismo, deficiencia de alfa-1-antitripsina y síndrome de Zellweger.

En la evaluación de un neonato con colestasis puede ser difícil distinguir SA de AB. Los niños con SA pueden presentar heces acólicas en forma persistente. La pobreza de conductos biliares está presente solo en el $60 \%$ de las biopsias hepáticas de niños con SA menores de 6 meses y la proliferación de conductos biliares es también un hallazgo frecuente en la infancia. Además, la hipoplasia del tracto biliar extrahepático se suele observar en el SA, lo cual complica la interpretación de la colangiografía intraoperatoria.

Otro aspecto digno de mención en el diagnóstico de SA es la presencia de una estenosis pulmonar periférica, más específica de él, si el paciente no padece síndrome de Williams, con el cual está también asociada.

La confirmación molecular está disponible comercialmente y para investigación. En este sentido, la mutación JAG1 fue encontrada en el $54 \%$ de los casos con tres y en el $34 \%$ con uno o dos hallazgos clínicos vinculados al SA..$^{8-11,29}$

\section{Tratamiento de la colestasis Médico}

Manejo médico intensivo del prurito y los xantomas, desde las primeras manifestaciones de la enfermedad, para evitar el deterioro de la calidad de vida. Tener en cuenta que muchos niños evolucionarán con mejoría de su colestasis.

Tratamiento conservador del prurito:

- Mantener la piel hidratada con productos emolientes, recortar las uñas y tomar baños breves. Nunca frotar con la toalla después del baño.

- Estimular el flujo biliar con coleréticos; el más utilizado es el ácido ursodesoxicólico. Se ha demostrado mejoría del prurito, xantomas y marcadores bioquímicos de colestasis. El fenobarbital se utiliza excepcionalmente, debido a su efecto sedante. La colesteramina, una resina quelante de los ácidos biliares, es eficaz pero difícil de administrar por su característica poco apetecible y por tener que ser dada $2 \mathrm{~h}$ alejada de otras medicaciones. Los antihistamínicos pueden proporcionar leve alivio sintomático. La rifampicina ha sido bien estudiada en el SA, obteniéndose buena respuesta en relación al prurito. La administración de naltrexona ha sido eficaz en adultos $y$, de acuerdo a experien- cias aisladas, lo sería también en la población pediátrica. La hipercolesterolemia no requiere tratamiento dietético ni medicamentoso. Las estatinas deben ser reservadas para aquellos casos con xantomas debilitantes. ${ }^{30,31}$

\section{Quirúrgico}

- Derivación biliar parcial externa o exclusión ileal: han resultado exitosas en algunos pacientes; constituyen opciones válidas en los casos con prurito intratable y xantomas. En pacientes con fibrosis hepática los resultados fueron pobres, por lo cual se aconseja la biopsia hepática para descartarla previa a su realización.

- Trasplante hepático: la indicación está centrada en los niños con cirrosis e insuficiencia hepática y puede discutirse en los pacientes con colestasis grave, prurito invalidante y pobre calidad de vida. La decisión de trasplantar un paciente con SA exige una minuciosa consideración, debido a su naturaleza multisistémica. La frecuencia elevada de complicaciones vasculares (trombosis) y sangrado en el pretrasplante y el postrasplante, deben ser tenidas en cuenta cuando se discute esta posibilidad. Desde un punto de vista pronóstico, el paciente con SA trasplantado tiene una menor sobrevida que la que se verifica para otras indicaciones electivas pediátricas. ${ }^{32,36}$

\section{Tratamiento de la afectación cardíaca}

Se han empleado con éxito técnicas invasivas no quirúrgicas, incluidas valvuloplastía, dilatación con balón e implantación de endotutor (stent). De ser necesario, los pacientes pueden ser sometidos a las técnicas quirúrgicas estándar, siendo su sobrevida inferior a la de los pacientes no SA con las mismas cardiopatías. El tratamiento de una cardiopatía congénita cianótica puede mejorar la función hepática, con reducción de la colestasis. $^{4,8-13}$

\section{Tratamiento del retraso del crecimiento y la malnutrición \\ Considerar las siguientes alternativas:}

- Debido a la malabsorción de ácidos grasos de cadena larga, suplementar las fórmulas con triglicéridos de cadena mediana.

- El aporte de hidratos de carbono puede mejorar el déficit calórico.

- Indicar sonda nasogástrica o gastrostomía para asegurar el aporte necesario. Esta última alternativa está contraindicada en los casos de cirrosis e hipertensión portal. 
- Las vitaminas liposolubles deben ser administradas individualmente, evitando los polivitamínicos por las dosis disímiles de sus componentes.

- Suplementación de calcio y zinc., ${ }^{4,-13}$

\section{Tratamiento de la afectación vascular}

No existen estudios prospectivos que informen sobre la naturaleza y prevalencia de las anomalías vasculares; por lo tanto, el diagnóstico de lesiones asintomáticas plantea un dilema ético. Cuando se presentan síntomas neurológicos es necesario realizar la evaluación correspondiente. Antes de indicar un trasplante hepático es aconsejable realizar un mapeo vascular del abdomen para identificar posibles anomalías. ${ }^{4,11}$

\section{Tratamiento de la afectación renal}

El compromiso renovascular sintomático puede ser tratado con la colocación de un endotutor (stent). La acidosis tubular renal mejora con la administración de bicarbonato. ${ }^{11,21,22}$

\section{BIBLIOGRAFÍA}

1. Watson GH, Miller V. Arterio-hepatic dysplasia. Familial pulmonary arterial stenosis with neonatal liver diseases. Arch Dis Child 1973;48:459-66.

2. Alagille D, Odièvre M, Gautier M, Dommergues JP. Hepatic ductular hypoplasia associated with characteristic facies, vertebral malformations, retarded physical, mental, and sexual development, and cardiac murmur. J Pediatr 1975;86:63-71.

3. Alagille D, Estrada A, Hadchouel M, Gautier M, et al. Syndromic paucity of interlobular bile ducts (Alagille syndrome or arteriohepatic dysplasia): review of 80 cases. J Pediat 1987;110:195-200.

4. Crosnier C, Lykavieris P, Meunier-Rotival M, Hadchouel $\mathrm{M}$. Alagille syndrome: the widening spectrum of arteriohepatic dysplasia. Clinics in Liver Disease 2000;4(4):765-78.

5. Oda T, Elkahloun AG, Pike BL, Okajima K, et al. Mutations in the human JAGGED1 gene are responsible for Alagille syndrome. Nat Genet 1997;16:235-42.

6. Li L, Krantz ID, Deng Y, Genin A, et al. Alagille syndrome is caused by mutations in human JAGGED1, which encodes a ligand for NOTCH1. Nature Genetics 1997;16(3):243-51.

7. Crosnier C, Driancourt C, Reynaud N, Dhorne-Pollet S, et al. Mutations in JAGGED1 gene are predominantly sporadic in Alagille syndrome. Gastroenterology 1999;116(5): 1141-8.

8. Emerick KM, Rand EB, Goldmuntz E, Krantz ID, et al. Features of Alagille syndrome in 92 patients: frequency and relation to prognosis. Hepatology 1999;29:822-29.

9. Lykavieris P, Hadchouel M, Chardot C and Bernard O. Outcome of liver disease in children with Alagille syndrome: a study of 163 patients. Gut 2001;49:431-35.

10. Subramaniam P, Knisely A, Portmann B, Qureshi SA, et al. Diagnosis of Alagille syndrome-25 years of experience at King's College Hospital. J Pediatr Gastroenterol Nutr 2011;52:84-9.

11. Tumpenny PD, Ellard S. Alagille syndrome: pathogenesis, diagnosis and management. Eur J Hum Genet 2012; 20(3):251-7.
12. McElhinney DB, Krantz ID, Bason L, Piccoli KM, et al: Analysis of cardiovascular phenotype and genotype-phenotype correlation in individuals with a JAG1 mutation and/or Alagille syndrome. Circulation 2002;106:2567-74.

13. Eldadah ZA, Hamosh A, Biery NJ, Montgomery RA, et al. Familial Tetralogy of Fallot caused by mutation in the JAGGED1 gene. Hum Mol Genet 2001;10:163-69.

14. Hingorani M, Nischal KK, Davies A, Bentley C, et al. Ocular abnormalities in Alagille syndrome. Ophthalmology 1999;106:330-37.

15. Sanderson E, Newman V, Haigh SF, Baker A, et al. Vertebral anomalies in children with Alagille syndrome: an analysis of 50 consecutive patients. Pediatr Radiol 2002;32:114-19.

16. Bales CB, Kamath BM, Munoz PS, Nguyen A, et al: Pathologic lower extremity fractures in children with Alagille syndrome. J Pediatr Gastroenterol Nutr 2010;51:66-70.

17. Olsen IE, Ittenbach RF, Rovner AJ, Leonard MB, et al: Deficits in size-adjusted bone mass in children with Alagille syndrome. J Pediatr Gastroenterol Nutr 2005;40:76-82.

18. Kamath BM, Loomes KM, Oakey RJ, Emerick KEM, et al. Facial features in Alagille syndrome: Specific or Alagille syndrome? Am J Med Genet 2002;112(2):163-70.

19. Emerick KM, Krantz ID, Kamath BM, Darling C, et al: Intracranial vascular abnormalities in patients with Alagille syndrome. J Pediatr Gastroenterol Nutr 2005;41(1): 99-107.

20. Lykavieris !, Crosnier C, Trichet C, Meunier-Rotival M, et al. Bleeding tendency in children with Alagille syndrome. Pediatrics 2003;111:167-70.

21. Shrivastava R, Williams A, Mikhail A, Roberts D, et al. An unusual cause of hypertension and renal failure: a case series of a family with Alagille syndrome. Nephron Dial Transplant 2010;25:1501-6.

22. Davis J, Griffiths R, Larkin K, Rozansky D, et al. Glomerular basement membrane lipidosis in Alagille syndrome. Pediatr Nephrol 2010;25:1181-84.

23. Bucuvalas JC, Horn JA, Carlsson L, Balistreri WF, et al. Growth hormone insensitivity associated with elevated circulating growth hormone-binding protein in children with Alagille syndrome and short stature. J Clin Endocr Metab 1993;76:1477-82.

24. Kamath BM, Bason L, Piccoli DA, Krantz ID, et al. Consequences of JAG1 mutations. J Med Genet 2003;40:891-95.

25. Spinner NB, Colliton RP, Crosnier C, Krantz ID, et al. JAGGED1 mutations in alagille syndrome. (Review). Hum Mutat 2001;17:18-33.

26. Colliton RP, Bason L, Lu FM, Piccoli DA, et al. Mutation analysis of JAGGED1 (JAG1) in Alagille syndrome patients. Hum Mutat 2001;17:151-52.

27. Hofmann JJ, Zovein AC, Koh H, Radtke F, et al. JAGGED1 in the portal vein mesenchyme regulates intrahepatic bile duct development: insights into Alagille syndrome. Development 2010;137:4061-72.

28. McDaniell R, Warthen DM, Sanchez-Lara PA, Pai A, et al. $\mathrm{NOTCH} 2$ mutations cause Alagille syndrome, a heterogeneous disorder of the NOTCH signaling pathway. Am J Hum Genet 2006;79(1):169-73.

29. Moyer V,Freese DK, Whitington PF, Olson AD, et al. Guideline for the Evaluation of Cholestatic Jaundice in Infants: Recommendations of the North American Society for Pediatric Gastroenterology, Hepatology and Nutrition. J Pediatr Gastroenterol Nutr 2004;39:115-28.

30. Mattei P, von Allmen D, Piccoli D, Rand E: Relief of intractable pruritus in Alagille syndrome by partial external biliary diversion. J Pediatr Surg 2006;41:104-7.

31. Kamath BM, Loomes KM, Piccoli DA. Medical management of Alagille syndrome. J Pediatr Gastroenterol Nutr 2010;50:580-6. 
32. Kaye AJ, Rand EB, Munoz PS, Spinner NB, et al. Effect of Kasai procedure on hepatic outcome in Alagille syndrome. J Pediatr Gastroenterol Nutr 2010;51:319-21.

33. Yang H, Porte RJ, Verkade HJ, De Langen ZJ, et al. Partial external biliary diversion in children with progressive familial intrahepatic cholestasis and Alagille disease. J Pediatr Gastroenterol Nutr 2009;49:216-21.

34. Rumbo C, Sandi M, Padín JM, Farinelli P, et al. Derivación biliar externa parcial: Alternativa terapéutica en el síndro- me de Alagille. Primer caso en Argentina. Acta Gastroenterol Latinoam 2011;41:324-30.

35. Kamath BM, Schwarz KB, Hadzic N: Alagille syndrome and liver transplantation. J Pediatr Gastroenterol Nutr 2010; 50:11-5.

36. Arnon R, Annunziato R, Miloh T, Suchy F, et al. Orthotopic liver transplantation for children with Alagille syndrome. Pediatr Transplantation 2010;14:622-8. 